\title{
Papel del prelímbico en la adquisición de la memoria de preferencia hacia estímulos asociados a la cocaina
}

\author{
ISIS GIL MIRAVET \\ al080291@uji.es \\ ZAIRA TORRES GARRIDO \\ al226805@uji.es \\ FERNANDO GONZÁLEZ HERNÁNDEZ \\ al228888@uji.es \\ Araceli Palma Gómez \\ al296129@uji.es \\ Marta Miquel Salgado-Araujo \\ miquel@uji.es
}

\section{Resumen}

Introducción: Hallazgos previos sugieren que el desarrollo de la adicción a las drogas puede ser entendido en términos de interacción entre los mecanismos cerebrales de memoria pavloviana e instrumental que subyacen a la búsqueda e ingesta de drogas. Se argumenta que el consumo de drogas es un comportamiento inicialmente dirigido a un objeto, pero después de la experiencia repetida puede convertirse en un hábito desencadenado automáticamente por los estímulos asociados al consumo. En este caso, uno de los procesos que subyace a este cambio de comportamiento es la adquisición de la memoria de preferencia hacia las señales ambientales asociadas a la droga. La evidencia apoya que la red prefronto-estriado-límbica sustenta el almacenamiento de estas memorias inducidas por drogas. Objetivo y métodos: El presente estudio tuvo como objetivo evaluar los efectos de la inactivación de la corteza prefrontal medial (prelímbica-infralímbica) (CPFm) en la adquisición de la preferencia hacia una señal de olor emparejada con la cocaína (Cs+). La inactivación del CPFm se llevó a cabo mediante la infusión de lidocaína en dicha región diez minutos antes del entrenamiento. Resultados: Los resultados indicaron que la inactivación de la CPFm aumentó hasta el $100 \%$ el porcentaje de animales que adquieren preferencia condicionada por la cocaína. Conclusiones: Nuestros resultados demuestran que el desarrollo de la preferencia condicionada inducida por la cocaína se promueve cuando la actividad del CPFm disminuye. Por lo tanto, estos resultados sugieren que la corteza prefrontal medial trabaja en la adquisición de la inhibición de memorias emocionales relacionadas con las drogas.

Palabras clave: cerebro, cocaína, neuroplasticidad. 


\begin{abstract}
Introduction: Previous findings suggest that the development of drug addiction can be understood in terms of interactions between Pavlovian and instrumental memory mechanisms in the brain that underlie drug seeking and taking. It is argued that drug use is a behavior initially goal-directed, but after repeated experience it can become a habit automatically triggered by drug-associated stimuli. In this case, one of the processes underlying this behavioral shift is the acquisition of preference memories towards drug-related environmental cues. Evidence supports that prefronto-striatallimbic networks underpin storage of these drug-induced memories. Aim and methods: The present study aimed at evaluating the effects of medial prefrontal cortex (MPFC) deactivations on the acquisition of preference towards an odour cue paired to cocaine (cs+). Medial PFC deactivation was accomplished by infusing lidocaine into the region ten minutes before training. Results: The results indicated that deactivation of MPFC increased up to $100 \%$ the percentage of animals acquiring conditioned preference for cocaine. Conclusions: Our findings demonstrated that the development of cocaine-induced conditioned preference is promoted when MPFC activity decreases. Therefore, these results suggest that the medial prefrontal cortex works on inhibiting acquisition of drug-related emotional memories.
\end{abstract}

Keywords: brain, cocaine, neuroplasticity.

\title{
Introducción
}

La adicción a los estimulantes psicomotores es un trastorno crónico recurrente, y la prevención de las recaídas es uno de los principales objetivos para su tratamiento (Mendelson y Mello, 1996). El éxito del tratamiento es difícil por la hipersensibilidad a las propiedades motivacionales de incentivo de los estímulos asociados al consumo de la droga (Robinson y Berridge, 1993). Uno de los procesos clave que va aparejado a la búsqueda de droga y a su consumo es el almacenamiento a largo plazo de los recuerdos relacionados con la droga, que contribuyen a orientar la respuesta a estímulos relacionados con ésta (Everitt y Robbins, 2005; Kalivas y Volkow, 2005; Hyman, Malenka y Nestler, 2006; Robinson y Berridge, 2008). Algunos estudios han sugerido que estas memorias asociadas a las señales relacionadas con la droga se almacenan y reactivan por las interacciones de dopamina y glutamato en el circuito prefrontestriatal (Belin y Everitt, 2008; Volkow y Wang, Tomasi y Baler, 2013).

La corteza prefrontal de los mamíferos está implicada en una amplia gama de funciones cognitivas que reflejan su importancia en la optimización y asignación de los recursos para satisfacer de forma flexible las cambiantes demandas de tareas y reglas (Euston, Gruber, y McNaughton, 2012). Su patrón de conectividad, de vías aferentes y eferentes, indica que la corteza prefrontal es esencial para integrar la información de los sistemas sensoriales, límbicos y vegetativos, para ejercer el control ejecutivo sobre funciones motoras, cognitivas y autonómicas a través de conexiones topográficas con el cuerpo estriado (Alexander y Crutcher, 1990; Groenewegen y Uylings, 2000; Chudasama y Robbins, 2006; Hoover y Vertes, 2007). Las regiones prelímbica $(\mathrm{PL})$ e infralímbica (IL) de la corteza prefrontal medial (CPFm) de la rata son componentes del bucle "afectivo" cortico-estriatal, que reciben entradas excitadoras del hipocampo y de la amígdala basolateral (Ishikawa y Nakamura, 2003). En diversos estudios se 
ha observado que el córtex prefrontal medial está implicado en el control contextual sobre la conducta motivada, siendo esta inducida por el restablecimiento del contexto de búsqueda de drogas (Peters, Kalivas, y Quirk, 2009; Bossert y cols., 2011).

La creciente evidencia pone de relieve la importancia de considerar las subregiones de la CPFm que parecer ser funcionalmente distintas, distinguiéndose entre la zona dorsal (cingulado anterior + PL) y la zona ventral (IL) (Ashwell y Ito, 2014). En concreto, el córtex prefrontal medial dorsal se ha relacionado con la inhibición del restablecimiento de la búsqueda de cocaína inducida por contexto, mientras que el aspecto ventral se ha asociado con la conducta dirigida de búsqueda de cocaína (Peters, LaLumiere, y Kalivas, 2008), aunque no todos los datos son consistentes con esta dicotomía propuesta (Bossert y cols., 2011). En conjunto, podría sugerirse que el PL-IL suministra un interruptor de encendido y apagado para la expresión de la conducta condicionada de búsqueda de drogas, sobre todo después de la extinción (Peters y cols., 2009).

En esta investigación, nos centramos en la corteza prefrontal medial, una región que parece ser punto clave dentro del circuito motivacional por el que una gran variedad de estímulos convergen para producir una respuesta de búsqueda de la droga. Principalmente, nos centraremos en las subregiones PL e IL, ya que ambas han sido abordadas anteriormente, tanto en los estudios de búsqueda de drogas mediante autoadministración (Shalev, Grimm y Shaham, 2002), como en estudios de restablecimiento de la respuesta de búsqueda de droga (McLaughlin y Ver, 2003; Rocha y Kalivas, 2010; Rogers, Ghee y See, 2008). En este modelo de condicionamiento pavloviano, en el que las unidades de motivación serán estímulos olfativos asociados a la cocaína, esperamos que estos estímulos ambientales fomenten la probabilidad de activar la respuesta de búsqueda de droga (Carbo-Gas y cols., 2014), viéndose esta aumentada al inactivar de manera temporal la CPFm.

\section{Método}

\section{Animales}

Se obtuvieron 16 ratas Sprague-Dawley macho con un peso de 200-250 gr (Janvier; St Berthevin Cedex, Francia). Las ratas fueron alojadas individualmente en jaulas con comida y agua ad libitum. La sala de estabulación se mantuvo a $22 \pm 2{ }^{\circ} \mathrm{C}$ con las luces encendidas de $8 \mathrm{~h}$ a $20 \mathrm{~h}$. Cuando las ratas tuvieron un peso de $270-300 \mathrm{gr}$ se les realizó una cirugía estereotáxica, y tras una semana de recuperación, comenzó el procedimiento experimental. El protocolo experimental se aplicó en las primeras $5 \mathrm{~h}$ del ciclo de luz. Todos los procedimientos con animales se realizaron de conformidad con la directiva del Consejo de la Comunidad Europea (86/609/CCE), Real Decreto BOE 34/11370/2013 y la directiva local de DOGV 26/2010.

\section{Fármacos}

La cocaína se administró por vía intraperitoneal (IP) y la lidocaína intracerebral. El clorhidrato de cocaína fue proporcionado por Alcaliber SA (Madrid, España) y el clorhidrato de lidocaína se adquirió en Sigma-Aldrich (Madrid, España). Todos los fármacos se disolvieron en solución salina al 0,9\%. La lidocaína al $6 \%$ se infundió 10 minutos antes de cada ensayo experimental. La cocaína $(15 \mathrm{mg} / \mathrm{kg} / \mathrm{ml})$ se inyectó inmediatamente antes de cada ensayo. La solución salina al $0,9 \%$ se utilizó como el vehículo control. 


\section{Cirugía}

A las 16 ratas se les practicó una cirugía estereotáxica. Los animales fueron anestesiados con clorhidrato de ketamina $(100 \mathrm{mg} / \mathrm{kg}$; IP) y clorhidrato de xilazina (10 mg/kg; IP). Las ratas fueron fijadas en un aparato estereotáxico, y se les implantó una cánula guía (calibre 22) justo en el CPFm (coordenadas: AP: 3,2 , ML: $\pm 0,6$, y DV $-3,5$ relativa al bregma, línea media, y la superficie del cráneo, respectivamente). A la mitad de las ratas se le implantó en el lado izquierdo y a la otra mitad en el derecho. Se fijo la cánula al cráneo mediante tres tornillos de acero inoxidable y cemento dental. Se insertó un estilete en cada cánula guía para evitar el bloqueo. Se dejó a las ratas una recuperación postquirúrgica de una semana para su completa recuperación.

\section{Microinyecciones}

Antes de cada ensayo, se retiraron los estiletes y se insertaron unos inyectores que mediante un tubo de polietileno estaban conectados a una bomba de infusión. Los inyectores eran $1 \mathrm{~mm}$ más largos que las cánulas, con la idea de que la infusión se realizara justo entre prelímbico e infralímbico, afectando a la zona del córtex prefrontal medial (CPFm) que nos interesaba. Se infundió un 1 microl en un periodo de $2 \mathrm{~min}$, dejando el inyecto en el interior por un tiempo de minuto y medio para una correcta difusión. Tras este periodo, se les retiraba el inyector y se les insertaba de nuevo el estilete. Las infusiones se realizaron $10 \mathrm{~min}$ antes de comenzar cada ensayo. A la mitad de los animales se les infundió lidocaína al $6 \%$ y a la otra mitad salina al $0,9 \%$

\section{Procedimiento comportamental y diseño experimental}

El procedimiento experimental se realizó en un corredor de metacrilato oscuro ( $90 \times 20 \times 40)$ con dos departamentos en sus extremos donde se confinaba a los animales durante $15 \mathrm{~min}$ al día.

Inicialmente se realizaron una pruebas de preferencia para comprobar que dos olores fueran igualmente preferidos (lavanda y rosas). Tras dicha comprobación, se utilizaron estos olores como estímulos para el estudio. Se depositaron tres gotas de lavanda o rosas en unos trozos de papel, que se presentaron dentro de unas bolas de acero con agujeros, que sobresalían en una de las paredes del brazo del corredor. Uno de los olores actuó como cs+ y se asoció a la cocaína (15 mg/kg, IP). En días alternos, las ratas fueron expuestas a la otra fragancia (cs-) junto a inyecciones de solución salina. Se llevaron a cabo un total de 8 sesiones de emparejamiento con cocaína utilizando un diseño ABAB. Los olores utilizados como cS+ y CS- se contrabalancearon entre los animales, al igual que los lados del corredor.

La preferencia se evaluó $48 \mathrm{~h}$ después de la última administración de cocaína en un test de 30 minutos sin droga, en el que los animales se colocaban en el centro del corredor y se les presentaban simultáneamente en brazos opuestos el CS+ y el CS. Las sesiones fueron grabadas en vídeo y se registró el tiempo (TS) que pasaban en cada extremo del corredor durante los últimos 20 minutos por un observador ciego. La preferencia se calculó como [TS en CS+ 1 (TS en CS+ + TS en CS-)] $\times 100$. 


\section{Resultados}

Mediante un ANOVA de un factor se comparó la preferencia por el cs+, entre el grupo control y el grupo con inactivación, observándose un efecto significativo para el grupo experimental (F1,14 = 9,212, $p<0,01)$ sobre el grupo Sham (figura 1).

Sin embargo, como se puede observar en el diagrama de dispersión (figura 2), no todos los sujetos del grupo Sham que recibieron inyecciones de cocaína emparejadas con el cs+ mostraron una puntuación de preferencia más alta que el punto de indiferencia (50\%). Por esta razón se utilizó una puntuación de preferencia de corte arbitrario del 60 \% para poder distinguir entre animales «condicionados» $(n=4)$ y «no condicionado» $(n=4)$. Posteriormente se realizaron comparaciones entre el grupo lidocaína, el grupo condicionado y el grupo no condicionado mediante un ANOVA de un factor. Se observó un efecto significativo del grupo experimental $(\mathrm{F} 2,13=9,831, p<0,01)$. Pruebas post-hoc revelaron que el grupo lidocaína mostró una mayor preferencia por el lado que contiene el cs+ que el grupo condicionado y el no condicionado ( $p<0,01$ en ambos casos) (figura 3).

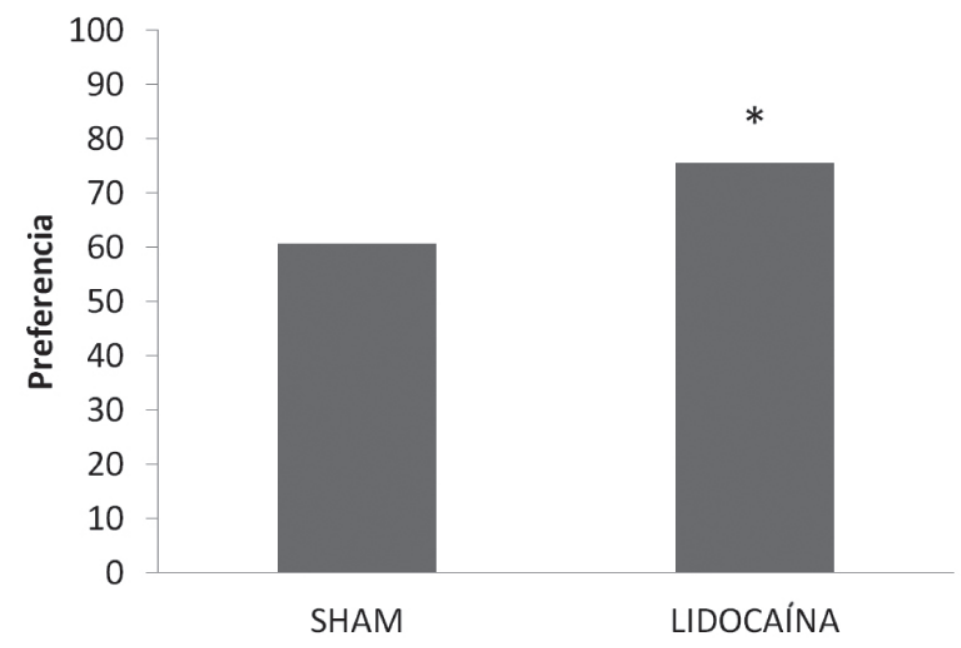

Figura 1. Comparación de la media del porcentaje del día del test entre el grupo Sham y el grupo lidocaína

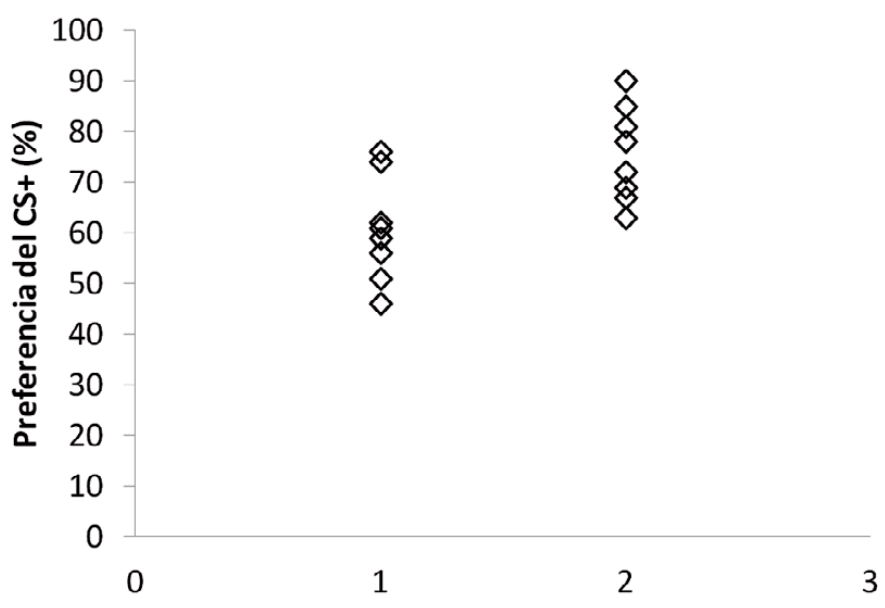

Figura 2. Diagrama de dispersión de la preferencia inducida por un olor asociado a la cocaína en el grupo Sham y el grupo lidocaína 


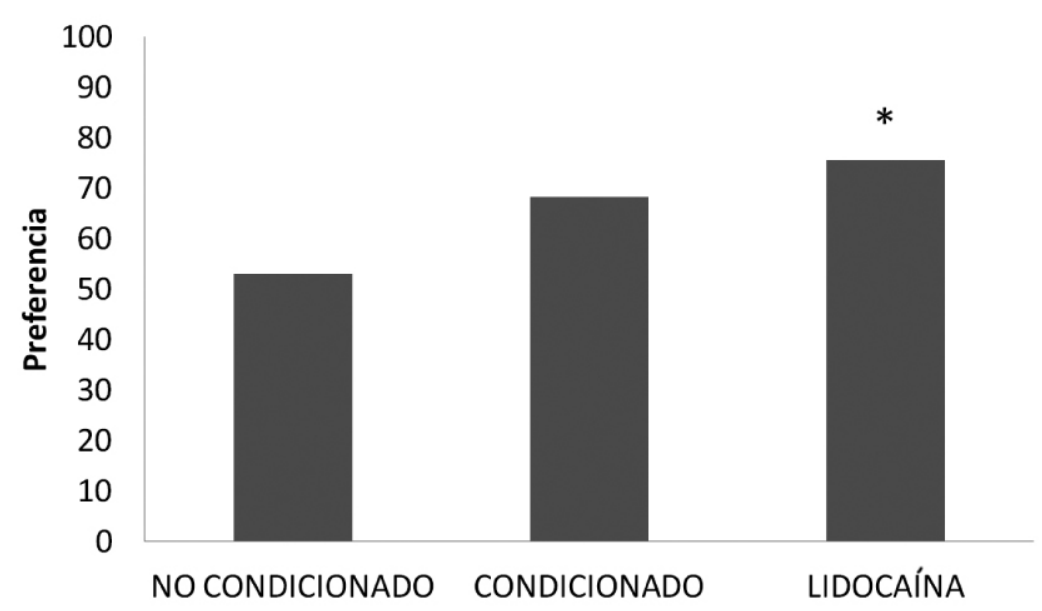

Figura 3. Preferencia del día del test inducida por un olor asociado a la cocaína dividiendo el grupo Sham en no condicionado y condicionado

\section{Discusión y conclusiones}

Tras el experimento los resultados obtenidos concuerdan con los ya adquiridos en experimentos anteriores en ratones (Carbo-Gas y cols., 2014), con porcentajes de preferencia por el estímulo que predice la disponibilidad de la cocaína similares en ambas especies. También, como decíamos en la introducción, se observa que la inactivación del CPFm produce una facilitación de la adquisición del condicionamiento inducido por cocaína que llega hasta el 100 \% de la muestra. Por tanto, la inactivación aumenta la probabilidad de adquirir la memoria inducida por la droga. Pero, además, la magnitud del condicionamiento parece incrementarse también, dado que el porcentaje de preferencia por el cs+ es superior en el grupo de inactivación que en el grupo Sham condicionado.

Estos resultados, junto a la literatura ya existente, podrían sugerir que el infralímbico toma el control en el bloqueo de las conductas dirigidas a la búsqueda de la droga, y que, por tanto, al bloquear ambas zonas (PL e IL), ésta se ve facilitada. De ser ciertas estas conclusiones, deben ser otras zonas del circuito prefronto-estriatal, probablemente más dorsales, las que actúen como facilitadoras de la conducta condicionada.

En cuanto a las limitaciones que hemos hallado, la no diferenciación de PL e IL a la hora de inactivar el CPFm podría ser un punto clave en la explicación de la preferencia. En diversos artículos se ha observado que el infralímbico es una pieza fundamental en el restablecimiento de la conducta motivada, el hecho de realizar el test $48 \mathrm{~h}$ después de la última presentación del cs+ emparejado a la cocaína, podríamos estar produciendo una extinción de respuesta de búsqueda de droga.

En posteriores investigaciones deberíamos centrarnos en desactivar ambas zonas por separado ( $\mathrm{PL}$ e IL) y hacer tanto hincapié en la adquisición de la conducta motivada, como en la extinción de la misma, observando las diferencias de ambas zonas y su influencia en cada uno de los ensayos. 


\section{Referencias bibliográficas}

Alexander, G. E., y Crutcher, M. D. (1990). Functional architecture of basal ganglia circuits: neural substrates of parallel processing. Trends in Neurosciences, 13, 266-71.

Ashwell, R., y Ito, R. (2014). Excitotoxic lesions of the infralimbic, but not prelimbic cortex facilitate reversal of appetitive discriminative context conditioning: the role of the infralimbic cortex in context generalization. Frontiers in Behavioral Neuroscience, 8, 63.

Ball, K. T., y Slane, M. (2012). Differential involvement of prelimbic and infralimbic medial prefrontal cortex in discrete cue-induced reinstatement of 3,4-methylenedioxymethamphetamine (MDMA; ecstasy) seeking in rats. Psychopharmacology, 224, 377-85.

Belin, D., y Everitt, B. J. (2008). Cocaine seeking habits depend upon dopamine-dependent serial connectivity linking the ventral with the dorsal striatum. Neuron, 57, 432-41.

Bossert, J. M., Stern, A. L., Theberge, F. R. M., Cifani, C., Koya, E., Hope, B. T., y Shaham, Y. (2011). Ventral medial prefrontal cortex neuronal ensembles mediate context-induced relapse to heroin. Nature Neuroscience, 14, 420-2.

Carbo-Gas, M., Vázquez-Sanroman, D., Gil-Miravet, I., De las Heras-Chanes, J., Coria-Ávila, G. A., Manzo, J., Miquel, M. (2014). Cerebellar hallmarks of conditioned preference for cocaine. Physiology and Behavior, 132, 24-35.

Chudasama, Y. y Robbins, T. W. (2006). Functions of frontostriatal systems in cognition: comparative neuropsychopharmacological studies in rats, monkeys and humans. Biological Psychology, 73, 19-38.

Euston, D. R., Gruber, A. J., y McNaughton, B. L. (2012). The role of medial prefrontal cortex in memory and decision making. Neuron, 76, 1057-70.

Everitt, B. J., y Robbins, T. W. (2005). Neural systems of reinforcement for drug addiction: from actions to habits to compulsion. Nature Neuroscience, $8,1481-9$.

Groenewegen, H. J., y Uylings, H. B. (2000). The prefrontal cortex and the integration of sensory, limbic and autonomic information. Progress in Brain Research, 126, 3-28.

Hoover, W. B., y Vertes, R. P. (2007). Anatomical analysis of afferent projections to the medial prefrontal cortex in the rat. Brain Structure and Function, 212, 149-79.

Hyman, S. E., Malenka, R. C., y Nestler, E. J. (2006). Neural mechanisms of addiction: the role of reward-related learning and memory. Annual Review of Neuroscience, 29, 565-98.

Ishikawa, A., y Nakamura, S. (2003). Convergence and Interaction of Hippocampal and Amygdalar Projections within the Prefrontal Cortex in the Rat. The Journal of Neuroscience, 23, 9987-9995.

Kalivas, P. W., y Volkow, N. D. (2005). The neural basis of addiction: a pathology of motivation and choice. The American Journal of Psychiatry, 162, 1403-13.

Mendelson, J. H., y Mello, N. K. (1996). Management of cocaine abuse and dependence. The New England Journal of Medicine, 334, 965-72.

Peters, J., Kalivas, P. W., y Quirk, G. J. (2009). Extinction circuits for fear and addiction overlap in prefrontal cortex. Learning \& Memory, 16, 279-88.

Peters, J., LaLumiere, R. T., y Kalivas, P. W. (2008). Infralimbic prefrontal cortex is responsible for inhibiting cocaine seeking in extinguished rats. The Journal of Neuroscience, 28, 6046-53.

Robinson, T. E., y Berridge, K. C. (1993). The neural basis of drug craving: an incentive-sensitization theory of addiction. Brain Research. Brain Research Reviews, 18, 247-91.

Robinson, T. E., y Berridge, K. C. (2008). Review. The incentive sensitization theory of addiction: some current issues. Philosophical Transactions of the Royal Society of London. Series B, Biological Sciences, 363, 3137-46. 
Rocha, A., y Kalivas, P. W. (2010). Role of the prefrontal cortex and nucleus accumbens in reinstating methamphetamine seeking. The European Journal of Neuroscience, 31, 903-9.

Rogers, J. L., Ghee, S., y See, R. E. (2008). The neural circuitry underlying reinstatement of heroin-seeking behavior in an animal model of relapse. Neuroscience, 151, 579-88.

Shalev, U., Grimm, J. W., y Shaham, Y. (2002). Neurobiology of relapse to heroin and cocaine seeking: a review. Pharmacological Reviews, 54(1), 1-42.

Volkow, N. D., Wang, G.-J., Tomasi, D., y Baler, R. D. (2013). Unbalanced neuronal circuits in addiction. Current Opinion in Neurobiology, 23, 639-48. 